\title{
THREE-LEGGED WALKING ROBOT ON A FRAGILE CYLINDER
}

\author{
Yury Filippovich Golubev \& Elena Vadimovna Melkumova
}
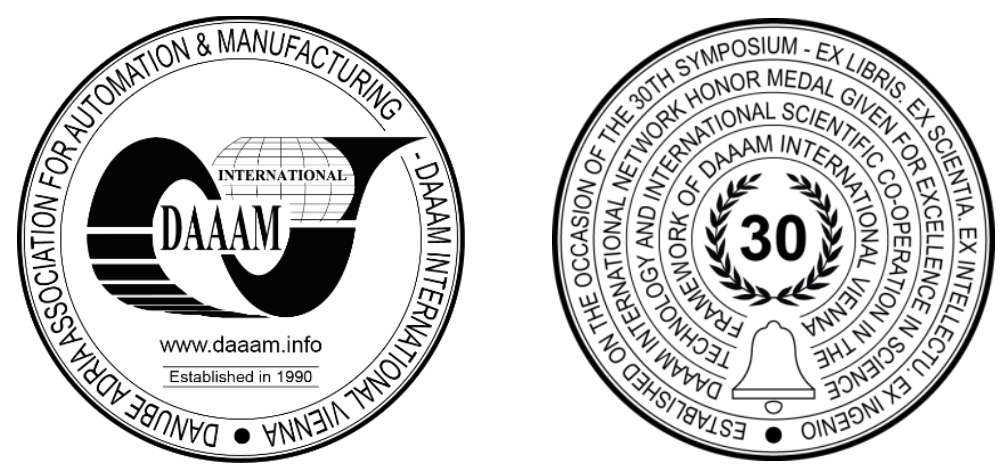

This Publication has to be referred as: Golubev, Y[ury] \& Melkumova, E[lena] V[adimovna] (2019). Three-legged Walking Robot on a Fragile cylinder, Proceedings of the 30th DAAAM International Symposium, pp.1181-1187, B. Katalinic (Ed.), Published by DAAAM International, ISBN 978-3-902734-22-8, ISSN 1726-9679, Vienna, Austria DOI: $10.2507 / 30$ th.daaam.proceedings.166

\begin{abstract}
We consider the walking robot with tree legs, where each leg contacts the surface of the fragile cylinder in a single foothold. Given the motion of the robot's legs with respect to its body, we solve the problem of finding the reaction forces, both analytically and numerically. The first step in solving similar problem belongs to N.Y.Zhukovsky. We describe the robot motion in terms of the general dynamics theorems, with six different equations of the robot's dynamics from the momentum and angular momentum theorems. In the special case a robot with three legs, the existence of the solution is related to a set of straightforward inequalities. Using numerical simulations we develop the classification of footholds positions for different values of the friction coefficient. This problem equivalent to the problem of curved object grasping by the fingers of the robot-manipulator. Each leg contacts the cylinder in a single supporting point with AmontonsCoulomb friction. There is an analogy of the equilibrium of a robot on a cylinder for the problems of transfer by a manipulator of a fragile cylinder or for robot which legs suspension points are on the cylinder. Two supporting points can be on one diameter in the cylinder base.
\end{abstract}

Keywords: Three-legged robot; Amontons-Coulomb friction; fragile cylinder.

\section{Introduction}

The problem of the distribution of bipedal reactions was studied in this work. For walking robot depending on the position of the fulcrum on the outer surface of the inclined rough non-holding cylinder taking into account reactions along its axis. This task arose as a development of similar studies to move the walking apparatus horizontally the plane [1].

The first step in the study of a similar problem was made N.E.Zhukovsky. In [2], he investigated equilibrium solid in the plane in the presence of friction.

Movement of a six-legged walking apparatus in quasistatic mode planes are investigated in the work of D.E.Okhotsimsky and Yu.F. Golubev [1], using the following criterion static stability of the apparatus. Walking apparatus, leaning o horizontal plane, statically stable in some moment of time if and only if the vertical projection of its center of mass on the supporting surface lies strictly inside the support polygon corresponding to this moment. A natural question arises, what do they look like similar criteria for other surfaces. Including criterion of non-rollover of the robot.

In the work [3] Yu.F. Golubev and I.G. Kolpakova using linear methods programmers have developed an iterative algorithm for distribution of reactions at randomly located points support walking machine. Later, F.L. Chernousko in work [4] cited a series of statements about the conditions of guaranteed equilibrium of the body on rough plane. Study of 
the organization of the movement of the octopus walking apparatus special design designed for movement in pipes in the presence of friction was carried out in the works [5], [6].

In [7] investigated the conditions of static stability walking robot on a perfect inner surface horizontal cylinder. In [8] the problem was considered for a rough horizontal cylinder, provided that there are no components of the reactions along the generatrix of the cylinder.

The proposed work continues the study begun by [9], where the problem was considered for a rough horizontal cylinder taking into account reactions along its axis.

The results of solving the equilibrium problem solid under the action of three forces applied to equilibrium biped walking robot on the outer surface of a rough inclined non-holding cylinder. The first contains the statement of the problem. In the second the conditions of equilibrium of a robot under the action of two forces in application to the equilibrium problem of a robot based only on one of the legs. The third is devoted to the balance of the robot, leaning on two legs. The fourth discusses two geometrical equilibrium conditions for a robot based on two legs on top hat.

In this paper, we consider the problem of curved object grasping by the fingers of the robot-manipulator. For example we discussed a three legged humanoid robot with pair of five arms fingers or a monkey-robot with twenty arms and legs fingers. The robot can hold the object by one and grasp by two fingers. An object grasping problem is equivalent to the problem of the walking robot with $n$ legs. Consider a grasp with $\mathrm{m}$ fingers. Each finger contacts an object in one foothold.

There is an analogy of this problem to the problem of walking robot dynamics on one-side constraint.

\section{Main result}

The kinetic moment of the robot relative to the point $O$ is considered. In order for the given motion to be realized, the reactions $\widetilde{\mathbf{R}}_{i}$ at $n$ support points must satisfy the following kinetostatic equations [1]:

$$
\sum_{i} \widetilde{\mathbf{R}}_{i}=-\widetilde{\boldsymbol{\Phi}}, \quad \sum_{i} \widetilde{\mathbf{r}}_{i} \times \widetilde{\mathbf{R}}_{i}=-\widetilde{\mathbf{M}}, \quad i=1, \ldots, n,
$$

where $\widetilde{\mathbf{R}}_{i}$ are the reaction components, $\widetilde{\mathbf{r}}_{i}$ are the radius vectors of the pivot points, $\widetilde{\boldsymbol{\Phi}}$ is the sum of the d'Alembert forces of inertia and external active forces, $\widetilde{\mathbf{M}}$ is the sum of the moments of d'Alembert inertia forces and the moments of external active forces relative to the fixed point $O$.

Assuming that $\widetilde{\boldsymbol{\Phi}}$ is orthogonal $\widetilde{\mathbf{M}}$, we obtain: [8] that the system $\{\widetilde{\boldsymbol{\Phi}}, \widetilde{\mathbf{M}}\}$ can be also used at the point $C$

$$
\tilde{\mathbf{r}}_{C} \times \widetilde{\boldsymbol{\Phi}}=\widetilde{\mathbf{M}}, \quad \mathbf{r}_{C}=-\frac{\widetilde{\mathbf{M}} \times \widetilde{\boldsymbol{\Phi}}}{\widetilde{\boldsymbol{\Phi}}^{2}}, \quad \widetilde{\Phi}=|\widetilde{\boldsymbol{\Phi}}|,
$$

where $\mathbf{r}_{C}$ is the vector $\mathbf{O C}$, and $C$ corresponds to point at which the resultant of the reactions is acting.

Further problem of reactions distribution $\widetilde{\mathbf{R}}_{i}$ in some fixed point of time is investigated by the proposal that force $\widetilde{\boldsymbol{\Phi}}$ is acting at the point $C$ and force moment there is zero. Motion equations (1) for finding reactions of walking robot body arms and legs prescribed motion can be transformed [10]:

$$
\sum_{i} \widetilde{\mathbf{R}}_{i}=-\widetilde{\boldsymbol{\Phi}}, \quad \sum_{i} \widetilde{\mathbf{r}}_{i} \times \widetilde{\mathbf{R}}_{i}=-\widetilde{\mathbf{r}}_{C} \times \widetilde{\boldsymbol{\Phi}}
$$

We restrict ourselves to the case when $\widetilde{\boldsymbol{\Phi}} \neq 0$. We bring the system $\{\widetilde{\boldsymbol{\Phi}}, \widetilde{\mathbf{M}}\}$ to the resultant at a point [2]. The point $C$ will also be the point of application of the resultant reactions. For example, a point $C$ may be the center of mass of a transported object.

Next, we consider the problem of the distribution of reactions $\widetilde{\mathbf{R}}_{i}$ along the fulcrum at some fixed point $C$.
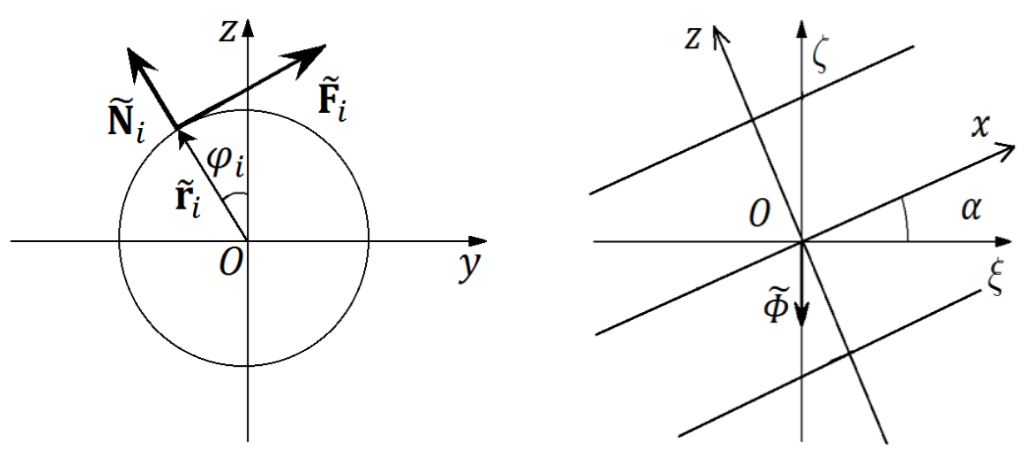

Fig. 1. Inclined cylinder. 
Let the robot support points be located on the surface of a rough straight circular cylinder with a dry friction coefficient $k$, Fig. 1 . And the axis of the cylinder is the angle $\alpha$ with the vector $\widetilde{\boldsymbol{\Phi}}$. In the case of two points of support, the model is supplemented by the introduction of the turning friction moment at the contact points. For three points of support, we restrict ourselves to the consideration of dry friction. The results apply to the case of sliding of support points with Amonton-Coulomb friction.

The kinetostatic equations are supplemented by inequalities responsible for getting reactions into the corresponding friction cones. The first group of inequalities is a consequence of the fact that the cylinder is an uncontrollable surface. When an object is captured internally, the scalar product of the reaction to the main normal to the surface at the fulcrum should be non-negative: $\widetilde{N}_{i}=\widetilde{\mathbf{R}}_{i} \cdot \mathbf{e}_{i}^{v} \geq 0$, where $\mathbf{e}_{i}^{v}$ is the external normal to the cylinder surface at the $i$-th fulcrum. With external capture, these inequalities are reversed.

Let write the vector $\boldsymbol{r}_{i}=\tilde{\mathbf{r}}_{i} / \rho=\left(x_{i}, y_{i}, z_{i}\right)$, in the cylinder coordinate: $\boldsymbol{r}_{i}=\left(x_{i},-\sin \varphi_{i}, \cos \varphi_{i}\right)$, normal $\mathbf{e}_{i}^{v}=$ $\left(0,-\sin \varphi_{i}, \cos \varphi_{i}\right)$, and the vector $\boldsymbol{N}_{i}=\widetilde{\mathbf{N}}_{i} / \widetilde{\Phi}=\left(0,-N_{i} \sin \varphi_{i}, N_{i} \cos \varphi_{i}\right)$, where $\varphi_{i}$ is the angles between axis $O z$ and cylinder normal $\mathbf{e}_{i}^{v}$. We define $\mathbf{e}_{x}$ as the unitary vector in the $O x$ axis, while the vector $\mathbf{e}_{i}^{\tau}=\left(0, \cos \varphi_{i}, \sin \varphi_{i}\right)$ as the tangential to the cylinder. Then the tangential reaction component: $\boldsymbol{F}_{i}=\tilde{\mathbf{F}}_{i} / \widetilde{\Phi}=\left(F_{i}^{x}, F_{i}^{y z} \cos \varphi_{i}, F_{i}^{y z} \sin \varphi_{i}\right)$, where $F_{i}^{x}=\boldsymbol{F}_{i} \cdot \mathbf{e}_{x}, F_{i}^{y z}=\boldsymbol{F}_{i} \cdot \mathbf{e}_{i}^{\tau}$, the reaction vector $\boldsymbol{R}_{i}=\widetilde{\mathbf{R}}_{i} / \widetilde{\Phi}=\left(R_{i}^{x}, R_{i}^{y}, R_{i}^{z}\right)$, center mass vector $\boldsymbol{r}_{C}=\widetilde{\mathbf{r}}_{C} / \rho=\left(x_{C}, y_{C}\right.$, $\left.z_{C}\right)$.

We further define the supporting points the coordinate differences along the cylinder, and angles difference: $\Delta x=$ $x_{2}-x_{1}, \Delta \varphi=\varphi_{2}-\varphi_{1}$. We then project system (2) onto the axes $O x y z$. For arbitrary surface we find that the second equation of (2) (corresponding to the angular moment) has the skew-symmetric matrix with respect to the component $R_{i}^{x}$ [11]. These are two independent equations, while the third equation corresponds to the restriction of the point $C$ to the plane containing the two supporting points. As a result, the system (2) yields five independent equation and a restriction:

$$
\begin{aligned}
& R_{1}^{x}=-R_{2}^{x}+\sin \alpha, R_{1}^{y}=-R_{2}^{y}, R_{1}^{z}=-R_{2}^{z}+\cos \alpha, \\
& \left(z_{1}-z_{2}\right) R_{1}^{x}+\left(x_{2}-x_{1}\right)\left(R_{1}^{z}-\cos \alpha\right)=\left(z_{C}-z_{2}\right) \sin \alpha \text {, } \\
& \left(y_{2}-y_{1}\right) R_{1}^{x}-\left(x_{2}-x_{1}\right) R_{1}^{y}=\left(y_{2}-y_{C}\right) \sin \alpha, \\
& \operatorname{tg} \alpha=\frac{\left(x_{2}-x_{1}\right)\left(\sin \varphi_{2}+y_{C}\right)+\left(x_{C}-x_{2}\right)\left(\sin \varphi_{2}-\sin \varphi_{1}\right)}{z_{c}\left(\sin \varphi_{2}-\sin \varphi_{1}\right)+\sin \left(\varphi_{1}-\varphi_{2}\right)+y_{C}\left(\cos \varphi_{2}-\cos \varphi_{1}\right)} . \\
& R_{1}^{x}=(p+\sin \alpha) / 2, \quad R_{2}^{x}=(\sin \alpha-p) / 2, \\
& R_{1}^{y}=\frac{p\left(y_{2}-y_{1}\right)}{2\left(x_{2}-x_{1}\right)}+\frac{2\left(y_{C}-y_{2}\right)+y_{2}-y_{1}}{2\left(x_{2}-x_{1}\right)} \sin \alpha, \\
& R_{2}^{y}=\frac{p\left(y_{1}-y_{2}\right)}{2\left(x_{2}-x_{1}\right)}+\frac{2\left(y_{2}-y_{C}\right)+y_{1}-y_{2}}{2\left(x_{2}-x_{1}\right)} \sin \alpha, \\
& R_{1}^{x}=\frac{2\left(x_{2}-x_{C}\right) \cos \alpha+\mathrm{p}\left(z_{2}-z_{1}\right)}{2\left(x_{2}-x_{1}\right)}+\frac{2\left(z_{C}-z_{2}\right)+z_{2}-z_{1}}{2\left(x_{2}-x_{1}\right)} \sin \alpha, \\
& R_{2}^{x}=\frac{2\left(x_{C}-x_{1}\right) \cos \alpha-\mathrm{p}\left(z_{2}-z_{1}\right)}{2\left(x_{2}-x_{1}\right)}+\frac{2\left(z_{2}-z_{C}\right)+z_{1}-z_{2}}{2\left(x_{2}-x_{1}\right)} \sin \alpha \\
& \operatorname{tg} \alpha=\frac{\left(x_{2}-x_{1}\right)\left(\sin \varphi_{2}+y_{C}\right)+\left(x_{C}-x_{2}\right)\left(\sin \varphi_{2}-\sin \varphi_{1}\right)}{z_{C}\left(\sin \varphi_{2}-\sin \varphi_{1}\right)+\sin \left(\varphi_{1}-\varphi_{2}\right)+y_{C}\left(\cos \varphi_{2}-\cos \varphi_{1}\right)} .
\end{aligned}
$$

If the number of contact points $n+m$ is even, and they are pairwise located at coincident points on opposite sides of a surface of almost zero thickness, then the indicated inequalities are satisfied.

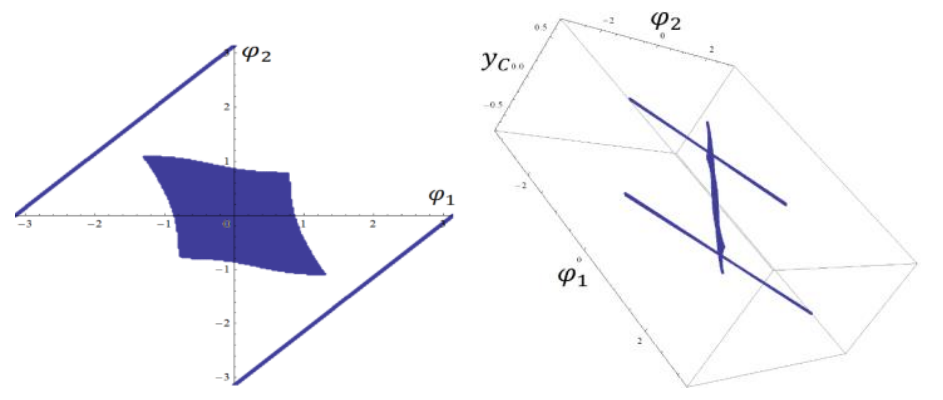

Fig. 2. Regions of parameters for which the problem of the distribution of reactions has a solution.

The second group of inequalities is the condition that the tangent components of the reactions are limited by the limiting value of the Coulomb dry friction. Those. kinetostatic [3] equations are supplemented by quadratic inequalities responsible for getting reactions into the corresponding friction cones. As soon as the modules of the tangent reactions exceed the limit value, slipping of the foot or finger relative to the surface occurs. 
These are quadratic inequalities with respect to the parameter $p$, where $p$ is the difference of the reaction components along the axis of the cylinder, $E$ which coincides and does not depend on $\alpha$ the coefficient $p$ of squared. Note that for an arbitrary surface, the structure and properties of these inequalities are preserved, Fig. 2.

When the robot moves, a change occurs in the one-support and two-support [4] phases. Let $\mathrm{n}=1$. The desired condition for the existence of motion is that the reaction at a single point of support must be equal to the force $\widetilde{\boldsymbol{\Phi}}$ modulo, and the reference point $C$ is located on a straight line along the force $\widetilde{\boldsymbol{\Phi}}$ passing through the point. Moreover, the slope of the line of action of the force $\widetilde{\boldsymbol{\Phi}}$ to the normal should not exceed the angle of friction.

Let $\mathrm{n}=2$. For the bi-support phase, the robot configurations are considered symmetrical with respect to point $\mathrm{C}$ along and across the axis of the cylinder. At $x_{2}=-x_{1}, \varphi_{2}=-\varphi_{1}$, for angles $\alpha=0$ and, $\alpha=\pi / 4$, below, fig. 3. charts are given for areas $E<0$ and $E \geq 0$. These graphs supplement and summarize the results obtained [5], [6].

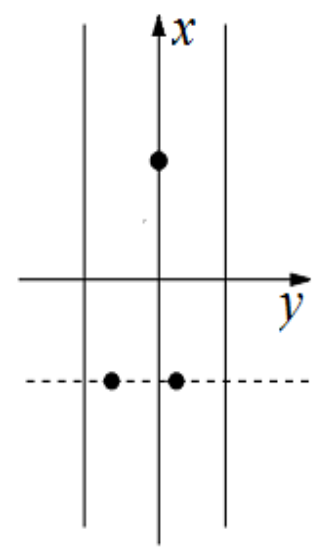

Fig. 3. Symmetrical along the axis of the cylinder configuration of the robot.

For robot finger configurations symmetrical about a point $\mathrm{C}$ along the axis of the cylinder, three cases with a non-negative coefficient $E$ are considered for the distance $x$ between the point $C$ and the reference points: $0,9,1$ and 1,1 for $\rho$ and $k$ equal 1, $\alpha$ from 0 to $\pi$ (all 14 different values of the angle of the cylinder) [7], [8]. Areas of existence of a solution to the problem of the distribution of reactions on the plane of two angles corresponding to the projections of the support points on the base of the cylinder and three-dimensional regions complementary to the indicated plane of the applicated point $C$ are constructed. When: $\alpha: 0, x: 1$ the area consists of three separately located connected subdomains. On the plane of the angles, each of a pair of parallel lines corresponds to a support in the diametrical plane of the cylinder containing the point $\mathrm{C}$. Between these lines is a connected area. It contains a straight line segment corresponding to equal angles, the robot rests from above, on a straight line parallel to the axis of the cylinder, satisfying the restriction on deviations from the direction of the force. The indicated segment on the graphs disappears when $x$ equal to 0,9 for $\alpha$ equal to $\pi / 4$, and when increasing $x$, later, when $4 \pi / 9$ [9], [10]. This corresponds to the fact that the cylinder begins to slide in the grip of the robot with two fulcrum. Valid areas for $\alpha=\pi / 3 ; x=1,1$ built in works [12], [13], [14].

We further define $\operatorname{s} \varphi_{21}=\sin \varphi_{2}-\sin \varphi_{1}, \mathrm{c} \varphi_{21}=\cos \varphi_{2}-\cos \varphi_{1}$. The conditions (4) can be displayed in the form:

$$
E p^{2}+B_{1} p+C_{1} \leq 0, \quad E p^{2}+B_{2} p+C_{2} \leq 0,
$$

where

$$
\begin{gathered}
E=\Delta x^{2}+\sin ^{2} \Delta \varphi-4 k^{2} \sin ^{4} \frac{\Delta \varphi}{2}, \\
B_{1}=4 \sin \frac{\Delta \varphi}{2}\left\{x_{2} \cos \alpha\left[\sin \varphi_{1} \cos \frac{\Delta \varphi}{2}-k^{2} \cos \varphi_{1} \sin \frac{\Delta \varphi_{2}}{2}\right]+B_{1}^{\alpha} \sin \Delta \varphi\right\}, \\
B_{2}=4 \sin \frac{\Delta \varphi}{2}\left\{x_{1} \cos \alpha\left[\sin \varphi_{2} \cos \frac{\Delta \varphi}{2}+k^{2} \cos \varphi_{2} \sin \frac{\Delta \varphi}{2}\right]+B_{2}^{\alpha} \sin \Delta \varphi\right\}, \\
C_{1}=x_{2}{ }^{2}\left[\sin ^{2} \varphi_{1}-k^{2} \cos ^{2} \varphi_{1}\right] \cos ^{2} \alpha+\left(x_{2}-x_{c}\right) \mathrm{C}_{11}^{\alpha} \sin 2 \alpha+\mathrm{C}_{12}^{\alpha} \sin \alpha^{2}, \\
C_{2}=x_{1}{ }^{2}\left[\sin ^{2} \varphi_{2}-k^{2} \cos ^{2} \varphi_{2}\right] \cos ^{2} \alpha+\left(x_{c}-x_{1}\right) \mathrm{C}_{21}^{\alpha} \sin 2 \alpha+\mathrm{C}_{22}^{\alpha} \sin \alpha^{2}, \\
B_{1}^{\alpha}=\cos \frac{\Delta \varphi}{2}\left(y_{c} \cos \varphi_{1}+z_{c} \sin \varphi_{1}\right)-k^{2} \sin \frac{\Delta \varphi}{2}\left(z_{c} \cos \varphi_{1}-y_{c} \sin \varphi_{1}-1\right)-2 \Delta x^{2}, \\
B_{2}^{\alpha}=\cos \frac{\Delta \varphi}{2}\left(\sin \Delta \varphi-y_{c} \cos \varphi_{2}-z_{c} \sin \varphi_{2}\right)+k^{2} \sin \frac{\Delta \varphi}{2}\left(y_{c} \sin \varphi_{2}-z_{c} \cos \varphi_{2}+\cos \Delta \varphi\right), \\
\mathrm{C}_{11}^{\alpha}=\left(y_{c} \cos \varphi_{1}+z_{c} \sin \varphi_{1}\right) \sin \varphi_{1}-k^{2}\left(z_{c} \cos \varphi_{1}-y_{c} \sin \varphi_{1}-1\right) \cos \varphi_{1}, \\
\mathrm{C}_{12}^{\alpha}=\left(y_{c} \cos \varphi_{1}+z_{c} \sin \varphi_{1}\right)^{2}-k^{2}\left(z_{c} \cos \varphi_{1}-y_{c} \sin \varphi_{1}-1\right)^{2}, \\
\mathrm{C}_{21}^{\alpha}=\left(\sin \Delta \varphi-z_{c} \sin \varphi_{2}-y_{c} \cos \varphi_{2}\right) \sin \varphi_{2}-k^{2}\left(y_{c} \sin \varphi_{2}-z_{c} \cos \varphi_{2}+\cos \Delta \varphi\right) \cos \varphi_{2}, \\
\mathrm{C}_{22}^{\alpha}=\left(\sin \Delta \varphi-z_{c} \sin \varphi_{2}-y_{c} \cos \varphi_{2}\right)^{2}-k^{2}\left(y_{c} \sin \varphi_{2}-z_{c} \cos \varphi_{2}+\cos \Delta \varphi\right)^{2} .
\end{gathered}
$$


The boundaries between different regimes can be determined analytically. For example, in the case of significant friction when $E<0$, the solution exists, and can be obtained analytically [10], as shown in Fig 2. Note that in this case it is limited to the range $x_{c}-x_{1} \leq 2 k \rho$. In contract to this behavior, for $E \geq 0$ there is no such restriction and an additional step is required to address the question of the existence of the solution. At the point $(0,0)$ we find: $E=0$, which means that two supportingpoints are orthogonal to the cylinder axis. Here, two possible solution are either identical, or limited to a single diameter. In the latter case, point $C$ and the reaction have to be in one plane, parallel to force $\widetilde{\boldsymbol{\Phi}}$, and the problem has a solution.

For the desired legs and arms configurations and given point $\mathrm{C}$, the problem can be solved numerically. In Fig 3 , we present the numerical solution for the example when $\alpha=0, x_{2}=-x_{1}=\rho==k=1$. Note that in this case $E>0$.

In the numerical simulations, we use a $300 \times 300$ array for the points $\left(\varphi_{1}, \varphi_{2}\right)$, in the interval $[-\pi, \pi]$, and for each point verify the conditions (8), (9). Specifically, the condition (9) was analyzed in two cases, when $E=0$ and $E>0$, and when the solution of the problem does exist, the solutions were shown in the plot.

When $E=0$, the reaction distribution problem reduces to the linear inequalities (8), (9) for the parameter $p$. To avoid zero division, signs of the coefficients in cycle were verified. For example if $B_{1}=0$, then necessary condition of the reaction distribution problem is $C_{1} \leq 0$.

For $E>0$, we need to consider two conditions. First is the restriction on the determinants $D_{i} \geq 0$, while the second is the requirement of a non-empty intersection of the set of points of the intervals between the roots of quadratic equations. If the point $\left(\varphi_{1}, \varphi_{2}\right)$ satisfies the conditions, then it shown on plot that shows the existing field of the reaction distribution problem. From this plot we see that, if two points are on one diameter, then the solution of the reaction distribution problem exists. The two lines in the plot, correspond to $\varphi_{1}=\varphi_{2}+\pi$ or $\varphi_{1}=\varphi_{2}-\pi$. The rhombus form represents the requirement on the determinants $D_{i} \geq 0$, while additional conditions further restrict the range. This is further illustrated in Fig 3, on the right, where the $3 D$-plot shows parameter range for the existence of the reactions distribution problem.

In Figs 4, 5 and 6, we present the results for $E>0$ and $E \leq 0$, when $x_{2}=-x_{1}, \varphi_{2}=-\varphi_{1}$. Fig 4 shows the case of $\alpha=0$, Fig. 5 corresponds to $\alpha=\pi / 4$, and Fig 6 describes the case when $\alpha$ increased to $\pi / 2$. Note that when $\alpha=\pi / 2$, the solution exists only for diametrical footholds.

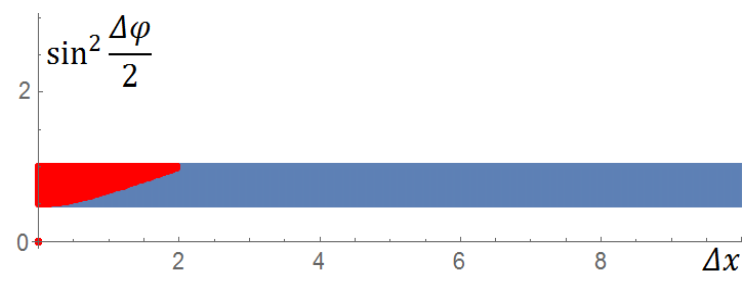

Fig. 4. For $\alpha$ increased to $\pi / 2 ; x_{2}=-x_{1}=\varphi_{2}=-\varphi_{1}$.

The boundaries between different regimes can be determined analytically. For two-legged robot when $E$ is negative, the solution exists, and obtained analytically. Using numerical simulations we explain the reaction distribution problem existing and build this problem solution existing fields for given supporting points and point $\mathrm{C}$ positions. For example, for two supporting points phase, we consider symmetric, about point $\mathrm{C}$, along and orthogonal cylinder axis, robot configurations. For first of these configurations examined three cases with nonnegative $E$ coefficient, for distance $x$, between point $C$ and footholds: 0,9; and 1,1 at $\rho$ and $k$ equal 1, $\alpha$ from 0 to $\pi$ (in all 13 different values cylinder inclination angles). Reactions distribution problem solution existing fields constructed on the two angles plane, corresponds to supporting points projections on the cylinder base and three dimensional fields which supplement this plane by point $C$ $z$-coordinate altitude. When $\alpha$ equals to $0, x$ equals to 1 , the field consist of three separate situated subregions. On the angle plane each of pair parallel lines corresponds to support on the cylinder diameter plane section contained point $\mathrm{C}$. There is connected field between these lines. It contains the line segment corresponding to the angles equality, robot supported above on the line which is parallel to cylinder axis and satisfy force direction deviation restriction. The indicated segment on the plot disappear when $x$ equals to 0,9 for $\alpha$ equals $\pi / 4$, and at increasing $x$, later, for $4 \pi / 9$. It corresponds to the robot beginning sliding down the cylinder. When $x$ equals to 1,1 for $\alpha$ equals $\pi / 3$ in three-dimensional fields observed bundles of separate points, Fig 7 . That means that the point $C$ altitude position more harsh change while changing the angles.

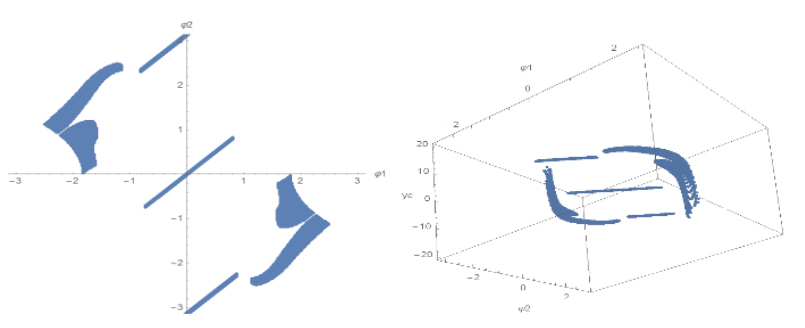

Fig. 5. Admissible areas for $\alpha=\pi / 3 ; \Delta x=1,1$. 
The results obtained in [4], [5] for a walking robot with two support points have an analogy for gripping with two support points of a holding cylinder.

\section{Conclusion}

During the robot motion, one-foothold and two-foothold phases are changed. Reaction distribution problem have a solution in following cases.

1. One foothold phases. So the motion existing condition is reaction is equal to force $\widetilde{\boldsymbol{\Phi}}$ and foothold and the point $C$ are on the line along $\widetilde{\boldsymbol{\Phi}}$. And the angle between $\widetilde{\boldsymbol{\Phi}}$ and the normal not exceed friction angle.

2. Two-foothold phases. The point $C$ and the reactions have to be in the plane parallel to force $\widetilde{\boldsymbol{\Phi}}$.

2.1. If footholds are on one diameter.

2.2. When the coefficient

$$
E=\Delta x^{2}+\sin ^{2} \Delta \varphi-4 k^{2} \sin ^{4} \frac{\Delta \varphi}{2}<0
$$

And in some fields with connected set of points, when $E \geq 0$.

So robot can move along the cylinder changing one and two-footholds phases.

3. Robot can hold the horizontal cylinder by three fingers. Or stay on it by three legs. Let one of the points is in vertical plane containing cylinder axis and another are in the plane orthogonal to the axis. Without friction, the cylinder center of mass has to be in the vertical plane that contain the cylinder axis. The supporting points are on the external surface of the lower semi-cylinder and the center mass of the cylinder is in the footholds triangle. If the first supporting point is in the lower semi-cylinder and two another are on the upper, the center of mass has to be out of the footholds triangle.

So the robot can transfer the cylinder by one, two or three fingers. Or stay on it by one, two or three legs. 4. And for example monkey-robot with 20 fingers or two legged human-robot with 10 arms fingers can hold the object by one and grasp by two-fingers.

About analogy to a grasp. Robot can hold the surface object by one finger. Or stay ob one leg. If the finger inside the cylinder, the center mass of an object is under the finger. If the finger out the cylinder, the center mass of an object is up the finger. And the angle between $\boldsymbol{P}$ and the normal not exceed friction angle.

The robot can hold an object with an arbitrary surface, such as a fragile cylinder, with one finger from the inside or outside, so that the center of mass of the surface is located, respectively, below or above the contact point. Moreover, the slope of the line of action of the force to the normal should not exceed the angle of friction. The grip can hold the cylinder with two fingers located on the same diameter. And with fingers located on opposite sides of the thin surface of the object at matching points.

\section{Acknowledgements}

Many thanks to colleagues N.N.Bolotnik, A.M.I.Krivtsov, E.I.Kugushev and V.E.Prynichnikov for advices.

\section{References}

[1] Zhukovsky, N. (1937). The condition for the equilibrium of a solid body resting on a fixed plane by some platform and able to move along this plane with friction. Complete Works, Vol. 1, - M.-L.: ONTI NKTP USSR Main Edition of Aviation Literature, PP. 433-449.

[2] Okhotsimsky, D. \& Golubev, Yu. (1984). Mechanics and motion control of an automatic walking apparatus. M .: Nauka, 312 p.

[3] Golubev, Yu. (2019). Fundamentals of Theoretical Mechanics. M .: Moscow State University, 728 p.

[4] Golubev, Yu. \& Melkumova, E. (1999.)Static stability conditions of a walking apparatus in a horizontal cylinder and on two planes // Bulletin of the Russian Academy of Sciences. Theory and control systems. No. 2. PP. 116-122.

[5] Melkumova, E. (2000). Oscillations of a walking apparatus around the axis of a smooth supporting cylinder // Moscow University Physics Bulletin. Series 1: Mathematics. Mechanics. No. 5. PP. 47-49.

[6] Golubev, Yu. \& Melkumova, E. (2010). Equilibrium of a biped robot on a rough horizontal cylinder, taking into account reactions along its axis. M.: MAKS Press, $64 \mathrm{p}$.

[7] Golubev, Yu. \& Melkumova, E. (2012). Walking Robot Dynamics on a Rough Inclined Cylinder. // ESMC - 8th European Solid Mechanics Conference. Graz, Austria, July 9-13, Book of Abstracts. Ed. Gerhard A. Holzapfel and Ray W. Ogden.

[8] Golubev, Yu. \& Melkumova, E. (2016). Prescribed Motion of a Two-legged Walking Robot on a Rough Cylinder. Proceedings of the International Conference "Stability and Oscillations of Nonlinear Control Systems" (Pyatnitskiy’s Conference), Moscow, V.A. Trapeznikov Institute of Control Sciences, PP. 1-4.

[9] Golubev, Yu. \& Melkumova, E. (2016). The existence of a given robot movement when using the pipe as a support. In the collection of the VII All-Russian meeting-seminar of the heads of departments and teachers of theoretical mechanics, robotics, mechatronics, universities of the Russian Federation. Materials of the meeting, Makhachkala: Publishing Center "Master", PP. 30-33. 
[10] Golubev, Yu. \& Melkumova, E. (2018). Two-legged Walking Robot Prescribed Motion On a Rough Cylinder // AIP Conference Proceedings, v. 1959, No. 030009, PP. 030009-1-030009-8.

[11] Golubev, Yu. \& Melkumova, E. (2018). An Analogy of the Equilibrium of a Two-legged Robot on a Cylinder for the Problem of Transfer by a Manipulator With a Two-finger Grasp of a Cylinder, APM, Proceedings of XLVI Summer School-Conf. "Advanced Problems in Mechanics", June 25-30, St. Petersburg, Russia; Polytech - IPME RAS, PP. 117-124, ISSN 2312-9921.

[12] Golubev, Yu. \& Melkumova, E. (2018). Footholds Admissible Areas Structure of a Two-legged Walking Robot on an Inclined Cylinder // Conference Series: Materials Science and Engineering, v. 468, No. 012003, PP. 1-9.

[13] Golubev, Yu. \& Melkumova, E. (2019). Brittle Cylinder Transfer by a Three-finger Grasp. In the collection of the VIII International Conference on Computational Methods for Coupled Problems in Science and Engineering (COUPLED), Sitges (Barcelona), Spain, June 3-5, Technical Program series, place of publication https://congress.cimne.com/coupled2019/admin/files/fileabstract/a374.pdf, abstract, p. 1-1.

[14] Golubev, Yu. \& Melkumova, E. (2019). Carrying a Fragile Cylinder with a Two-finger Grip Manipulator. In the collection Modern problems of aerohydrodynamics: Abstracts of the XIX All-Russian school-seminar dedicated to the 60th anniversary of the Scientific and Research Institute of Mechanics of Lomonosov Moscow State University. September 5-15, Sochi, "Burevestnik", Moscow University Publishing House Moscow, p. 39-39, Editors Afanasyev A., Makarova M., Chaika A. 